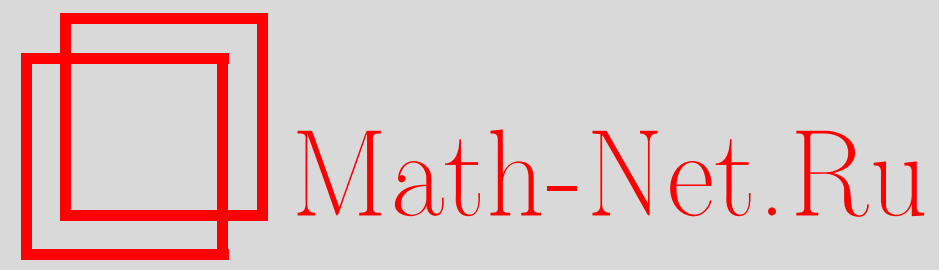

Н. П. Салихов, Об абсолютном критерии значимости для полиномиального распределения, Теория вероятн. и ее примен., 1997, том 42, выпуск 4, 731-746

DOI: https://doi.org/10.4213/tvp2182

Использование Общероссийского математического портала Math-Net.Ru подразумевает, что вы прочитали и согласны с пользовательским соглашением http://www . mathnet.ru/rus/agreement

Параметры загрузки:

IP : 54.162 .85 .209

26 апреля 2023 г., 13:34:29

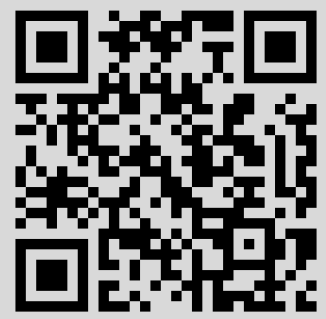


(C) 1997 г.

САЛИХОВ Н. П.*

\section{ОБ АБСОЛЮТНОМ КРИТЕРИИ ЗНАЧИМОСТИ ДЛЯ ПОЛИНОМИАЛЬНОГО РАСПРЕДЕЛЕНИЯ}

Найдены верхние оценки для суммы вероятностей всех тех событий $\left(x_{1}, \ldots, x_{r}\right)$, где $x_{k}$ - частота $k$-го исхода при $n$ независимых испытаниях по полиномиальной схеме испытаний $\mathrm{c} r$.возможными исходами, вероятность каждого из которых не превосходит вероятности события, наблюдаемого при $n$ независимых испытаниях по той же схеме. На этой основе построен критерий отклонения полиномиальной схемы при известных вероятностях исходов.

Ключевые слова и фразы: полиномиальная схема испытаний, абсолютный критерий значимости, расстояние Кульбака-Лейблера, состоятельность критерия при простой альтернативе, выпуклое программирование.

1. Введение. Рассмотрим полиномиальную схему $H(\mathbf{p})$ независимых испытаний, в которой $r \geqslant 2$ различных исходов появляются $c$ положительными вероятностями $p_{1}, \ldots, p_{r}, \mathbf{p}=\left(p_{1}, \ldots, p_{r}\right)>(0, \ldots, 0)$, $p_{1}+\cdots+p_{r}=1$. Пусть $\mathbf{n}=\left(n_{1}, \ldots, n_{r}\right)-$ событие, состоящее в том, что при $n$ испытаниях $k$-й исход появился $n_{k}$ раз, $n_{1}+\cdots+n_{r}=n$, $\Omega(n, r)$ - множество всех возможных событий $\mathbf{n}$. Упорядочим все $N=$ $C_{n+r-1}^{n}=|\Omega(n, r)|$ событий $\mathbf{n}$ в порядке невозрастания вероятностей

$$
\mathbf{P}(\mathbf{n})=\frac{n !}{n_{1} ! \cdots n_{r} !} p_{1}^{n_{1}} \cdots p_{r}^{n_{r}}: \mathbf{P}\left(\mathbf{n}_{1}\right) \geqslant \cdots \geqslant \mathbf{P}\left(\mathbf{n}_{N}\right) .
$$

Гипотезу $H(\mathbf{p}, \mathbf{n})$, состоящую в том, что событие $\mathbf{n} \in \Omega(n, r)$ появилось в результате $n$ независимых испытаний по схеме $H(\mathbf{p})$, естественно отклонить, когда сумма $\mathbf{P}_{\mathbf{n}}$ вероятностей всех событий $\mathbf{n}_{t}$, каждое из которых имеет вероятность не большую, чем вероятность наблюдаемого события $\mathbf{n}$, не превосходит малого числа $\varepsilon_{n} \in(0,1)$ :

$$
\mathbf{P}_{\mathbf{n}}=\sum_{\mathbf{P}\left(\mathbf{n}_{t}\right) \leqslant \mathbf{P}(\mathbf{n})} \mathbf{P}\left(\mathbf{n}_{t}\right) \leqslant \varepsilon_{n} .
$$

Такой в некотором смысле идеальный критерий значимости был введен К. Пирсоном в 1900 году. Он является примером абсолютного критерия значимости простой гипотезы (см. $[1$, с. 33$])$.

*ФАПСИ, Б. Кисельный пер., 4, 103031 Москва, Россия. 
Нашей целью будет разработка способов вычисления по наблюдаемому событию $\mathbf{n}$ верхней оценки вероятности $\mathbf{P}_{\mathbf{n}}$.

В п. 2 для наблюдаемого $\mathbf{n}$ определена статистика $\lambda_{n}$ и предложен критерий: гипотеза $H(\mathbf{p}, \mathbf{n})$ отклоняется, если статистика $\lambda_{n}$ удовлетворяет неравенствам $\lambda_{n}>0, \lambda_{n} \geqslant n^{-1}\left(\ln f\left(n, r, \mathbf{p}, \lambda_{n}\right)-\ln \varepsilon_{n}\right)$, где $f(n, r, \mathbf{p}, \lambda)$ - некоторая функция. При отклонении гипотезы $H(\mathbf{p}, \mathbf{n})$ этим критерием для наблюдаемого $\mathbf{n}$ выполняется оценка (1). Пункт 3 посвящен описанию множества событий $\mathbf{n}$, для которых $\lambda_{n}>0$. В п. 4 предложены варианты функции $f(n, r, \mathbf{p}, \lambda)$. В п. 5 полученные в пा. 2 , 4 оценки вида (1) сравниваются между собой и с известными асимптотическими оценками вероятности $\mathbf{P}_{\mathbf{n}}$. В пा. 6 показана состоятельность критерия п. 2 при альтернативной гипотезе $H(\mathbf{q}, \mathbf{n}), \mathbf{q} \neq \mathbf{p}$.

Далее будут использоваться следующие обозначения. В формулах будем полагать $a \ln b=0, a^{b}=1$ при $a=b=0$. Для наблюдаемого события $\mathbf{n}$ и для любого $\mathrm{x}=\left(x_{1}, \ldots, x_{r}\right) \in \Omega(n, r)$ определим векторы относительных частот:

$$
\underline{\mathbf{n}}=\left(\underline{n}_{1}, \ldots, \underline{n}_{r}\right)=\left(\frac{n_{1}}{n}, \ldots, \frac{n_{r}}{n}\right), \quad \underline{\mathbf{x}}=\left(\underline{x}_{1}, \ldots, \underline{x}_{r}\right)=\left(\frac{x_{1}}{n}, \ldots, \frac{x_{r}}{n}\right) .
$$

Введем следующие величины, функции и множества: $I(A)$ - индикатор события $A ; s=\min (n, r), p=\min _{1 \leqslant i \leqslant r} p_{i}$;

$$
I(\mathbf{a}, \mathbf{b})=\sum_{k=1}^{r} a_{k} \ln \left(\frac{a_{k}}{b_{k}}\right)
$$

- расстояние Кульбака-ЛІейблера от $\mathbf{a}=\left(a_{1}, \ldots, a_{r}\right) \geqslant(0, \ldots, 0)$ до $\mathbf{b}=\left(b_{1}, \ldots, b_{r}\right)>(0, \ldots, 0) ; L(0)=1, \varphi(0)=0, L(t)=t^{t}(t !)^{-1}, \varphi(t)=$ $t-\ln L(t)$ для натуральных $t$,

$$
\begin{aligned}
M(n, r) & =\max _{\mathbf{x} \in \Omega(n, r)} \sum_{k=1}^{r} \varphi\left(x_{k}\right) ; \\
\Omega(\mathbf{p}, \lambda) & =\left\{\mathbf{x}=\left(x_{1}, \ldots, x_{r}\right) \in \Omega(n, r): I(\underline{\mathbf{x}}, \mathbf{p}) \geqslant \lambda\right\}, \\
\omega(n, r) & =\left\{\mathbf{x}=\left(x_{1}, \ldots, x_{r}\right) \in \Omega(n, r): x_{1} \geqslant 1, \ldots, x_{r} \geqslant 1\right\} .
\end{aligned}
$$

\section{2. Верхняя оценка вероятности $\mathbf{P}_{\mathbf{n}}$.}

Теорема 1. Пусть функция $f(n, r, \mathbf{p}, \lambda)$ удовлетворяет при каждом $\lambda>0$ неравенству

$$
\Sigma(\lambda)=(L(n))^{-1} \sum_{\mathbf{x} \in \Omega(\mathbf{p}, \lambda)} \prod_{k=1}^{r} L\left(x_{k}\right) \leqslant f(n, r, \mathbf{p}, \lambda),
$$

a статистика $\lambda_{n}=I(\underline{\mathbf{n}}, \mathbf{p})-n^{-1}\left(M(n, r)-\sum_{k=1}^{r} \varphi\left(n_{k}\right)\right)$ удовлетворяет неравенствам $\lambda_{n}>0$,

$$
\lambda_{n} \geqslant n^{-1}\left(\ln f\left(n, r, \mathbf{p}, \lambda_{n}\right)-\ln \varepsilon_{n}\right)
$$


при некотором $\varepsilon_{n} \in(0,1)$. Тогда

$$
\mathbf{P}_{\mathbf{n}} \leqslant f\left(n, r, \mathbf{p}, \lambda_{n}\right) \exp \left(-n \lambda_{n}\right) \leqslant \varepsilon_{n} .
$$

Доказ а те ль с т в о. Неравенство $\mathbf{P}(\mathbf{x}) \leqslant \mathbf{P}(\mathbf{n})$ при $\mathbf{x}, \mathbf{n} \in$ $\Omega(n, r)$ равносильно неравенству

$$
I(\underline{\mathbf{x}}, \mathbf{p}) \geqslant I(\underline{\mathbf{n}}, \mathbf{p})-n^{-1}\left(\sum_{k=1}^{r} \varphi\left(x_{k}\right)-\sum_{k=1}^{r} \varphi\left(n_{k}\right)\right) .
$$

Следовательно, $\mathbf{P}_{\mathbf{n}} \leqslant \sum_{\mathbf{x} \in \Omega\left(\mathbf{p}, \lambda_{n}\right)} \mathbf{P}(\mathbf{x})$. Используя известное тождество

$$
\mathbf{P}(\mathbf{x})=(L(n))^{-1} \prod_{k=1}^{r} L\left(x_{k}\right) \exp (-n I(\underline{\mathbf{x}}, \mathbf{p}))
$$

(см., например, [2]), найдем, что

$$
\mathbf{P}_{\mathbf{n}} \leqslant \Sigma\left(\lambda_{n}\right) \exp \left(-n \lambda_{n}\right) \leqslant f\left(n, r, \mathbf{p}, \lambda_{n}\right) \exp \left(-n \lambda_{n}\right) .
$$

Равносильность неравенства $f\left(n, r, \mathbf{p}, \lambda_{n}\right) \exp \left(-n \lambda_{n}\right) \leqslant \varepsilon_{n}$ неравенству (2) доказывает теорему.

Вычислим величину $M(n, r)$, входящую в выражение для $\lambda_{n}$.

Теорема 2. Ecлu $n \leqslant r$, mо $M(n, r)=n$; еслu $n \geqslant r$, mo $M(n, r)=$ $r \varphi(m)+(n-r m)\left(1-m \ln \left(1+m^{-1}\right)\right)$, zде $m=[n / r]$.

I ока за т е ль с т в о. Докажем, что $\max _{\mathbf{x} \in \Omega(n, r)} \sum_{k=1}^{r} \varphi\left(x_{k}\right)=$ $M(n, r)$ достигается в таких точках $\mathbf{x}=\left(x_{1}, \ldots, x_{r}\right) \in \Omega(n, r)$, для кото-

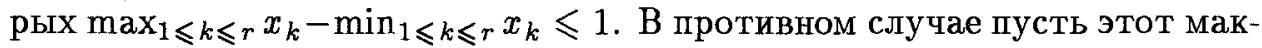
симум достигается в точке $\mathrm{x}=\left(x_{1}, \ldots, x_{r}\right) \in \Omega(n, r)$, в которой $x_{i}=a$, $x_{j}=b$, причем $a \geqslant b+2$. Рассмотрим точку $\mathbf{y}=\left(y_{1}, \ldots, y_{r}\right)$, в которой $y_{i}=a-1, y_{j}=b+1$, остальные координаты $y_{t}=x_{t}$. Тогда неравенство

$$
\sum_{k=1}^{r} \varphi\left(y_{k}\right)>\sum_{k=1}^{r} \varphi\left(x_{k}\right)
$$

равносильно неравенству $\left(1+(a-1)^{-1}\right)^{a-1}>1$ при $b=0$ и неравенству $\left(1+(a-1)^{-1}\right)^{a-1}>\left(1+b^{-1}\right)^{b}$ при $b \geqslant 1$. Так как функция $\left(1+x^{-1}\right)^{x}$ возрастает с ростом $x$ при $x>0$, то неравенство (3) доказано. Таким образом, $\max _{\mathbf{x} \in \Omega(n, r)} \sum_{k=1}^{r} \varphi\left(x_{k}\right)$ может достигаться лишь на множестве таких точек $\mathrm{x} \in \Omega(n, r)$, что $\max _{1 \leqslant k \leqslant r} x_{k}-\min _{1 \leqslant k \leqslant r} x_{k} \leqslant 1$. Если $n \leqslant r$, то указанные точки имеют $n$ координат $x_{i}=1$ и $r-n$ координат $x_{i}=0$. Для таких точек $\sum_{k=1}^{r} \varphi\left(x_{k}\right)=n \varphi(1)=n=M(n, r)$. Если $n \geqslant r$, то указанные точки имеют $n-r m$ координат $x_{i}=m+1$ и $r-(n-r m)$ координат $x_{i}=m$. Для таких точек

$$
\begin{aligned}
\sum_{k=1}^{r} \varphi\left(x_{k}\right) & =(n-r m) \varphi(m+1)+(r-n+r m) \varphi(m) \\
& =r \varphi(m)+(n-r m)\left(1-m \ln \left(1+m^{-1}\right)\right)=M(n, r) .
\end{aligned}
$$


3 а м е ч а н и е 1. С использованием неравенств для факториала из $[3$, с. 73$]$ получаются оценки

$$
\begin{aligned}
(12 t+1)^{-1} & <\varphi(t)-\ln (\sqrt{2 \pi t})<(12 t)^{-1} \\
0 & \leqslant I(\underline{\mathbf{n}}, \mathbf{p})-\lambda_{n} \leqslant h(\mathbf{n}), \\
h(\mathbf{n}) & =n^{-1}\left(c_{n}-\sum_{k=1}^{r}\left(\ln \left(\sqrt{2 \pi n_{k}}\right)+\left(12 n_{k}+1\right)^{-1}\right)\right),
\end{aligned}
$$

где $c_{n}=n$ при $n \leqslant r, c_{n}=r\left(\ln (\sqrt{2 \pi m})+(12 m)^{-1}\right)+(n-r m) \times$ $\left(1-m \ln \left(1+m^{-1}\right)\right), m=[n / r]$ при $n>r$. Отсюда следует, что

$$
\lim _{n \rightarrow \infty}\left(I(\underline{\mathbf{n}}, \mathbf{p})-\lambda_{n}\right)=0 .
$$

3 а м е ч а н и е 2. Если функция $f(n, r, \mathbf{p}, \lambda)$ не возрастает с ростом $\lambda$ при $\lambda>0$, то утверждение теоремы 1 справедливо для любой нижней ощенки статистики $\lambda_{n}$. В частности, можно заменить $\lambda_{n}$ величиной $I(\underline{\mathbf{n}}, \mathbf{p})-h(\mathbf{n})$.

3. О возможности применения теоремы 1. Найдем, для каких событий $\mathbf{n}$ выполняется условие $\lambda_{n}>0$ теоремы 1 . Неравенство $\lambda_{n}>0$ равносильно неравенству

$$
n+\sum_{k=1}^{r} \ln \left(n_{k} !\right)-\sum_{k=1}^{r} n_{k} \ln \left(n p_{k}\right)-M(n, r)>0,
$$

которое с учетом теоремы 2 преобразуется к неравенствам:

$$
P(\mathbf{n})< \begin{cases}\frac{n !}{(1 !)^{n}}\left(\frac{1}{n}\right)^{n}, & \text { если } n<r, \\ \frac{n !}{(m !)^{r}}\left(\frac{1}{r}\right)^{n}, & \text { если } n=m r, \\ \frac{n !}{((m+1) !)^{k}(m !)^{r-k}}\left(\frac{1}{r}\right)^{n} \theta_{n}, & \text { если } n=m r+k, 1 \leqslant k<r,\end{cases}
$$

где $\theta_{n}=(r m)^{m(r-k)}(r m+r)^{k(m+1)} / n^{n}$. Полагая $y=n /(r-k)>z=$ $(n-k) / r$, найдем, что

$$
\theta_{n}=\left(\left(1+\frac{1}{y}\right)^{y}\left(1+\frac{1}{z}\right)^{-z}\right)^{r-k}>1
$$

Таким образом, неравенство $\lambda_{n}>0$ выполняется по крайней мере для таких событий $\mathbf{n}$, вероятность $\mathbf{P}(\mathbf{n})$ которых меньше максимальной вероятности $n$ независимых испытаний по полиномиальной схеме с $s$ равновероятными исходами. Множество таких $\mathbf{n}$ не пусто, так как содержит 
событие, в котором исход с вероятностью $p$ встречается $n$ раз. Если $p_{1}=\cdots=p_{r}=1 / r$, то $\lambda_{n}>0$ всегда за исключением случая, когда $n=r m$ (при этом $\left.\lambda_{n}=n^{-1}\left(\sum_{k=1}^{r} \ln \left(n_{k} !\right)-r \ln m !\right)\right)$ и $n_{1}=\cdots=n_{r}=m$.

4. Варианты функций $f(n, r, \mathbf{p}, \lambda)$.

Лемма 1. Пусть $k, n$ - натуральные числа числа, $n \geqslant k u ф у н к-$ ция $g(x)$ выпукла на $[1, n-k+1]($ при $n>k)$.

Тогда при любом $\mathbf{x}=\left(x_{1}, \ldots, x_{k}\right) \in \omega(n, k)$

$$
k g\left(\frac{n}{k}\right) \leqslant \sum_{t=1}^{k} g\left(x_{t}\right) \leqslant g(n-k+1)+(k-1) g(1) .
$$

Доказательств о. Достаточно рассмотреть случай $n>$ $k>1$. Нижняя оценка следует из неравенства Йенсена. График функции $g(x)$ на $[1, n-k+1]$ не проходит выше прямой, соединяющей точки $(1, g(1)),(n-k+1, g(n-k+1))$. Следовательно,

$$
g\left(x_{i}\right) \leqslant \frac{n-k+1-x_{i}}{n-k} g(1)+\frac{x_{i}-1}{n-k} g(n-k+1)
$$

для $i=1, \ldots, k$. Суммируя эти неравенства, получим верхнюю ощенку.

Теорема 3. Условию (*) теоремы 1 удовлетворяют функции

$$
\begin{array}{ll}
f(n, r, \mathbf{p}, \lambda)=r\left(\frac{n+r}{n+1}\right)^{n-1}, & \text { если } 1 \leqslant n \leqslant 3, \\
f(n, r, \mathbf{p}, \lambda)=\left(\frac{n+r}{r}\right)^{r-1}, \quad \text { eсли } n \geqslant \frac{r}{w}, \\
f(n, r, \mathbf{p}, \lambda)=\frac{r}{n+r}\left(\frac{n+r}{n} h\right)^{n}, & \text { если } 4 \leqslant n<\frac{r}{w},
\end{array}
$$

где $w=0.25500097 \ldots$ единственный на интервале $(0,1)$ корень уравнения $(x+1) \ln \left(1+x^{-1}\right)=2$,

$$
h=(w+1)^{w-1} w^{-w}=1.196307 \ldots .
$$

Д ок а з а те л ь с т в о. Ощеним сверху величину

$$
\Sigma(0)=(L(n))^{-1} \Sigma
$$

где

$$
\begin{aligned}
\Sigma & =\sum_{\mathbf{x} \in \Omega(n, r)} \prod_{t=1}^{r} L\left(x_{t}\right)=\sum_{\mathbf{y} \in \omega(n+r, r)} \prod_{t=1}^{r} \frac{\left(y_{t}-1\right)^{y_{t}-1}}{\left(y_{t}-1\right) !} \\
& =\sum_{\mathbf{y} \in \omega(n+r, r)} \prod_{t=1}^{r} \frac{y_{t}^{y_{t}-1}}{y_{t} !} \Pi_{r}(y), \quad \Pi_{r}(y)=\prod_{t=1}^{r} y_{t}\left(\frac{y_{t}-1}{y_{t}}\right)^{y_{t}-1}
\end{aligned}
$$


Оценим сверху величину $\Pi_{r}(\mathbf{y})$ на множестве $\omega(n+r, r)$. Рассмотрим точки $\mathbf{y} \in \omega(n+r, r)$, в которых $r-k$ координат $y_{i}=1, k$ координат $y_{i} \geqslant$ $2, k \in\{1, \ldots, s\}$. На таких точках $\Pi_{r}(\mathbf{y})=\Pi_{k}(\mathbf{x})$, где $\mathbf{x}=\left(x_{1}, \ldots, x_{k}\right)$, $x_{1}+\cdots+x_{k}=n+k, x_{1} \geqslant 2, \ldots, x_{k} \geqslant 2$. Из представления $\Pi_{k}(\mathbf{x})=$ $\exp \left(-\sum_{i=1}^{k} g\left(z_{i}\right)\right)$, где $z_{i}=x_{i}-1$ для $i=1, \ldots, k, g(x)=(x-1) \ln (x+$ 1) $-x \ln x$, и леммы 1 следует, что

$$
\Pi_{k}(\mathbf{x}) \leqslant \exp \left(-k g\left(\frac{n}{k}\right)\right)=\left(\frac{n+k}{k}\right)^{k}\left(\frac{n}{n+k}\right)^{n}=q(k) .
$$

Положим $q=\max _{1 \leqslant k \leqslant s} q(k)$. Так как функция $q(k)$ имеет на $(0, n]$ единственный максимум в точке $w n$, то

$$
\begin{array}{lll}
q=q(1)=(n+1)^{1-n} n^{n}, & \text { если } & 1 \leqslant n \leqslant 3, \\
q=q(r)=\left(\frac{n+r}{r}\right)^{r}\left(\frac{n}{n+r}\right)^{n}, & \text { если } & n \geqslant \frac{r}{w}, \\
q=q(w n)=h^{n}, & \text { если } & 4 \leqslant n<\frac{r}{w} .
\end{array}
$$

Используя известное тождество

$$
\sum_{\mathrm{y} \in \omega(n+r, r)} \prod_{t=1}^{r} \frac{y_{t}^{y_{t}-1}}{y_{t} !}=r \frac{(n+r)^{n-1}}{n !}
$$

(см. $[4$, с. 181$],[5$, с. 48]), получаем из (4) оценку

$$
\Sigma(0) \leqslant \frac{n !}{n^{n}} q r \frac{(n+r)^{n-1}}{n !}=q r \frac{(n+r)^{n-1}}{n^{n}},
$$

что с учетом формул для $q$ доказывает теорему.

Теоремы 1, 3 дают возможность получить верхнюю ощенку для величины $\mathbf{P}_{\mathbf{n}} \exp \left(n \lambda_{n}\right)$, не зависящую от $\mathbf{p}, \lambda$. Найдем верхние оценки для этой величины, зависящие от $\mathbf{p}, \lambda$.

Рассмотрим случай, когда $r=2, p=p_{1} \leqslant p_{2}=1-p$. Пусть $I(x, p)=x \ln (x / p)+(1-x) \ln ((1-x) /(1-p))$. Тогда

$$
\mathbf{P}_{\mathbf{n}} \leqslant \sum_{I(k / n, p) \geqslant \lambda_{n}} C_{n}^{k} p^{k}(1-p)^{n-k}
$$

Отсюда с использованием оценок для биномиальных сумм из $[6$, c. $300-$ 301], получим:

$$
\begin{aligned}
\mathbf{P}_{\mathbf{n}} & \leqslant \sum_{k=n \beta}^{n} C_{n}^{k} p^{k}(1-p)^{n-k} \leqslant \frac{1-p}{\beta-p} \sqrt{\frac{\beta}{2 \pi(1-\beta) n}} \exp (-n I(\beta, p)) \\
& \leqslant \frac{1-p}{\beta-p} \sqrt{\frac{\beta}{1-\beta}} \frac{1}{\sqrt{2 \pi n}} \exp \left(-n \lambda_{n}\right)
\end{aligned}
$$




$$
\begin{aligned}
& \text { если }-\ln (1-p)<\lambda_{n} \leqslant-\ln p \\
& \begin{aligned}
\mathbf{P}_{\mathbf{n}} \leqslant & \sum_{k=0}^{n \alpha} C_{n}^{k} p^{k}(1-p)^{n-k}+\sum_{k=n \beta}^{n} C_{n}^{k} p^{k}(1-p)^{n-k} \\
\leqslant & \frac{p}{p-\alpha} \sqrt{\frac{1-\alpha}{2 \pi \alpha n}} \exp (-n I(\alpha, p)) \\
& +\frac{1-p}{\beta-p} \sqrt{\frac{\beta}{2 \pi(1-\beta) n}} \exp (-n I(\beta, p)) \\
\leqslant & \left(\frac{p}{p-\alpha} \sqrt{\frac{1-\alpha}{\alpha}}+\frac{1-p}{\beta-p} \sqrt{\frac{\beta}{1-\beta}}\right) \frac{1}{\sqrt{2 \pi n}} \exp \left(-n \lambda_{n}\right),
\end{aligned}
\end{aligned}
$$

если $0<\lambda_{n} \leqslant-\ln (1-p)$, где $n \alpha=\left[n \theta_{1}\right], n \beta-$ ближайшее целое число не меньшее $n \theta_{2}, \theta_{1}, \theta_{2}$ - корни уравнения $I(x, p)=\lambda_{n}, \theta_{1}<p<\theta_{2}$. Этот метод (даюший асимптотически наилучшую ощенку, см. п. 5) не удалось обобщить на случай $r>2$. Вместо этого укажем один метод получения в теореме 1 оценок $f(n, r, \mathrm{p}, \lambda)$ величины $\Sigma(\lambda)$, зависяших от всех четырех артументов, который при малых $\lambda_{n}$ ухудшает асимптотическую ощенку в $C n$ раз (константа $C$ неизвестна).

Лемма 2. Для величины $T(n, k)=\sum_{\mathbf{x} \in \omega(n, k)} \prod_{i=1}^{k} x_{i}^{-1 / 2}$ верна оченка $T(n, k)<\pi(2 \sqrt{n-k+1}-1)^{k-2}$, если $n \geqslant k \geqslant 2$.

Д ок а за т ел ь с т в. При. $k=n$ имеем $T(n, n)=1<\pi$. ІІри $n=2 m \geqslant 4$

$$
\begin{aligned}
2^{-1} T(n, 2) & =\frac{1}{n}+\sum_{i=1}^{m-1}(i(n-i))^{-1 / 2} \\
& \leqslant \frac{1}{n}+(n-1)^{-1 / 2}+\int_{1}^{m-1}(x(n-x))^{-1 / 2} d x \\
& =\frac{1}{n}+(n-1)^{-1 / 2}+\arcsin \left(1-\frac{2}{n}\right)-\arcsin \left(\frac{2}{n}\right)
\end{aligned}
$$

При $n=2 m+1 \geqslant 3$ аналогично имеем

$$
\begin{aligned}
2^{-1} T(n, 2) & =\sum_{i=1}^{m}(i(n-i))^{-1 / 2} \leqslant(n-1)^{-1 / 2}+\int_{1}^{m}(x(n-x))^{-1 / 2} d x \\
& =(n-1)^{-1 / 2}+\arcsin \left(1-\frac{2}{n}\right)-\arcsin \left(\frac{1}{n}\right) .
\end{aligned}
$$

Из этих формул с учетом неравенства $x-\arcsin (2 x)<0$ при $x \in\left(0, \frac{1}{4}\right]$ и возрастания функции $(x-1)^{-1 / 2}+\arcsin (1-2 / x)$ при $x>2$ следует оценка $T(n, 2)<2(n-1)^{-1 / 2}+2 \arcsin (1-2 / n) \leqslant 2 \arcsin 1=\pi$. Пусть 
доказано, что $T(n, m)<\pi(2 \sqrt{n-m+1}-1)^{m-2}$ при некотором $m \geqslant 2$ и всех $n \geqslant m$. Тогда при $n \geqslant m+1$

$$
\begin{aligned}
T(n, m+1) & =\sum_{i=1}^{n-m} i^{-1 / 2} T(n-i, m) \\
& <\pi \sum_{i=1}^{n-m} i^{-1 / 2}(2 \sqrt{n-i-m+1}-1)^{m-2} \\
& \leqslant \pi(2 \sqrt{n-m}-1)^{m-2} \sum_{i=1}^{n-m} i^{-1 / 2} \\
& \leqslant \pi(2 \sqrt{n-m}-1)^{m-2}\left(1+\int_{1}^{n-m} x^{-1 / 2} d x\right) \\
& =\pi(2 \sqrt{n-m}-1)^{m-1},
\end{aligned}
$$

что доказывает лемму.

Лемма 3. Пусть $k \in\{1, \ldots, s\}$, величина $\lambda>\gamma_{k}=n^{-1}(n-k+$ 1) $\ln (n-k+1)-\ln (n p)$, натуральные $i_{1}, \ldots, i_{k}$ таковы, что $1 \leqslant i_{1}<$ $\cdots<i_{k} \leqslant r$. Тогда каждое из $C_{r}^{k}$ множеств

$$
\Omega\left(\mathbf{p}, \lambda, i_{1}, \ldots, i_{k}\right)=\left\{\mathbf{x}=\left(x_{1}, \ldots, x_{r}\right) \in \Omega(\mathbf{p}, \lambda):\left(x_{i_{1}}, \ldots, x_{i_{k}}\right) \in \omega(n, k)\right\}
$$

nycmo.

Д ок а з а т е л ь с т в о. Пусть $\Omega\left(\mathbf{p}, \lambda, i_{1}, \ldots, i_{k}\right)$ не пусто. Тогда при некотором $\left(x_{i_{1}}, \ldots, x_{i_{k}}\right) \in \omega(n, k)$ имеем:

$$
\sum_{t=1}^{k} \underline{x}_{i_{t}} \ln \left(p^{-1} \underline{x}_{i_{t}}\right) \geqslant \lambda, \quad \sum_{t=1}^{k} x_{i_{t}} \ln \left(x_{i_{t}}\right) \geqslant n(\lambda+\ln (n p)) .
$$

Применяя лемму 1 , получим отсюда неравенства

$$
(n-k+1) \ln (n-k+1) \geqslant n(\lambda+\ln (n p)), \quad \lambda \leqslant \gamma_{k} .
$$

Заметим, что $0 \leqslant \gamma_{s}<\gamma_{s-1}<\cdots<\gamma_{2}<\gamma_{1}=-\ln p$, при этом $\gamma_{s}=0$ только в случае $n=r, p=1 / r$. Действительно, если $n<r$, то $s=n$, $\gamma_{s}=\gamma_{n}=-\ln (n p)>0$. Если же $n \geqslant r$, то $s=r, \gamma_{s}=\gamma_{r}=\gamma(n)$, где

$$
\begin{aligned}
\gamma(x) & =x^{-1}(x-r+1) \ln (x-r+1)-\ln (x p), \\
\gamma^{\prime}(x) & =x^{-2}(r-1) \ln (x-r+1)>0 \quad \text { для } x>r .
\end{aligned}
$$

Следовательно, $\gamma_{r}>\gamma(r)=-\ln (r p) \geqslant 0$ при $n>r, \gamma_{r}=\gamma(r)>0$,. при $n=r, p<1 / r$. Заметим также, что при $\lambda>\gamma_{1}$ множество $\Omega(\mathbf{p}, \lambda)$ пусто.

Теорема 4. Пусть при некотором $\sigma \in\{1, \ldots, s\}$ либо $\gamma_{\sigma+1}<\lambda \leqslant$ $\gamma_{\sigma}$, если $\sigma \neq s$, либо $0 \leqslant \lambda \leqslant \gamma_{s}$, если $\sigma=s$. 
Тогда условию (*) теоремы 1 удовлетворяют функиии

$$
\begin{gathered}
f(n, r, \mathbf{p}, \lambda)=\rho=\sum_{i=1}^{r} I\left(\lambda \leqslant-\ln p_{i}\right), \quad \text { если } \sigma=1, \\
f(n, r, \mathbf{p}, \lambda)=\rho+\frac{n ! e^{n}}{n^{n}}\left(\frac{r(r-1)}{4} \exp \left(-\frac{2}{6 n+1}\right)+\pi b_{n}\right), \\
\text { если } \sigma \geqslant 2,
\end{gathered}
$$

где $b_{n}=0$, если $\sigma=2$,

$$
\begin{aligned}
b_{n} & =y_{n} \frac{(2 \sqrt{n-1}-1)^{3}}{(2 \sqrt{n-2}-1)^{5}} Q(n, r, \sigma), \\
Q(n, r, \sigma) & =\sum_{k=3}^{\sigma} C_{r}^{k} g_{n}^{k}, \quad g_{n}=\frac{z_{n}}{\sqrt{2 \pi}} \frac{(2 \sqrt{n-2}-1)^{2}}{2 \sqrt{n-1}-1}, \\
y_{n} & =\exp \left(\frac{12 n}{(6 n+1)(4 n+1)}\right), \\
z_{n} & =\exp \left(-\frac{10 n+1}{(6 n+1)(4 n+1)}\right), \quad \text { если } \sigma \geqslant 3 .
\end{aligned}
$$

Д ок а з а т е л ь с т в о. Аналогично формуле (4) получим

$$
\Sigma(\lambda)=(L(n))^{-1} \Sigma_{1}(\lambda),
$$

где

$$
\Sigma_{1}(\lambda)=\sum_{\mathbf{x} \in \Omega(\mathbf{p}, \lambda)} \prod_{i=1}^{\tau} L\left(x_{i}\right)
$$

Представляя $\Omega(\mathbf{p}, \lambda)$ в виде суммы $C_{r}^{1}+C_{r}^{2}+\cdots+C_{r}^{s}$ попарно непересекаюшихся множеств $\Omega\left(\mathbf{p}, \lambda, i_{1}, \ldots, i_{k}\right)\left(1 \leqslant i_{1}<\cdots<i_{k} \leqslant r, k \in\right.$ $\{1, \ldots, s\})$ и замечая, что по лемме $3 \Omega\left(\mathbf{p}, \lambda, i_{1}\right)$ пусто, когда $-\ln p_{i_{1}}<\lambda$, а $\Omega\left(\mathbf{p}, \lambda, i_{1}, \ldots, i_{k}\right)$ пусто, когда $k \geqslant \sigma+1, \sigma \neq s$, получим: $\Sigma_{1}(\lambda)=\rho L(n)$, если $\sigma=1$,

$$
\Sigma_{1}(\lambda)=\rho L(n)+\sum_{k=2}^{\sigma} \sum_{1 \leqslant i_{1}<\cdots<i_{k} \leqslant r} \sum_{x \in \Omega\left(\mathbf{p}, \lambda, i_{1}, \ldots, i_{k}\right)} \prod_{i=1}^{r} L\left(x_{i}\right)
$$

если $\sigma \geqslant 2$. Используя оценку $n ! \geqslant \sqrt{2 \pi n}(n / e)^{n} \exp (1 /(12 n+1))$ $([3$, c. 73$])$, получим при $\sigma \geqslant 2$ оценку

$\Sigma_{1}(\lambda) \leqslant \rho L(n)+e^{n} \sum_{k=2}^{\sigma} C_{r}^{k} g^{k} \sum_{\mathbf{x} \in \omega(n, k)}\left(x_{1} \times \cdots \times x_{k}\right)^{-1 / 2} \exp \left(-\sum_{i=1}^{k} \frac{1}{12 x_{i}+1}\right)$, 
где $g=1 / \sqrt{2 \pi}$. Применяя лемму 1 к функции $(12 x+1)^{-1}$, получим оценку

$$
\Sigma_{1}(\lambda) \leqslant \rho L(n)+e^{n} \sum_{k=2}^{\sigma} C_{\tau}^{k} g^{k} \exp \left(-\frac{k^{2}}{12 n+k}\right) T(n, k),
$$

затем с помощью леммы 2 неравенство

$$
\Sigma_{1}(\lambda)<\rho L(n)+\pi e^{n} \sum_{k=2}^{\sigma} C_{r}^{k} g^{k} \exp \left(-\frac{k^{2}}{12 n+k}\right)(2 \sqrt{n-k+1}-1)^{k-2}
$$

При $\sigma=2$ отсюда следует оценка

$$
\Sigma_{1}(\lambda)<\rho L(n)+e^{n} \frac{r(r-1)}{4} \exp \left(-\frac{2}{6 n+1}\right) .
$$

Пусть $\sigma \geqslant 3, a_{k}=(2 \sqrt{n-k+1}-1) \exp \left(-k^{2} /(12 n+k)\right)$. Тогда

$$
\Sigma_{1}(\lambda)<\rho L(n)+e^{n} \frac{r(r-1)}{4} \exp \left(-\frac{2}{6 n+1}\right)+\pi e^{n} \Sigma_{2},
$$

где

$$
\begin{aligned}
\Sigma_{2} & =\sum_{k=3}^{\sigma} C_{\tau}^{k} g^{k}(2 \sqrt{n-2}-1)^{k-3} a_{k} \\
& =a_{2} \sum_{k=3}^{\sigma} C_{r}^{k} g^{k}(2 \sqrt{n-2}-1)^{k-3} \prod_{i=3}^{k}\left(\frac{a_{i}}{a_{i-1}}\right) \\
& \leqslant a_{2} \sum_{k=3}^{\sigma} C_{r}^{k} g^{k}(2 \sqrt{n-2}-1)^{k-3} \mu^{k-2} \quad \text { при } \mu=\max _{3 \leqslant \text { a }}\left(\frac{a_{i}}{a_{i-1}}\right)
\end{aligned}
$$

Итак, полагая $g_{n}=g(2 \sqrt{n-2}-1) \mu$, получим оценку

$$
\Sigma_{2} \leqslant \frac{a_{2}}{\mu^{2}(2 \sqrt{n-2}-1)^{3}} \sum_{k=3}^{\sigma} C_{r}^{k} g_{n}^{k}
$$

Докажем, что

$$
\mu=\frac{a_{3}}{a_{2}}=\frac{2 \sqrt{n-2}-1}{2 \sqrt{n-1}-1} z_{n} .
$$

Это верно при $\sigma=3$. При $\sigma>3$ достаточно доказать, что $a_{k+1} / a_{k}<$ $a_{k} / a_{k-1}$ для $k=3, \ldots, \sigma-1$, что равносильно неравенствам $\ln a_{k+1}+$ $\ln a_{k-1}<2 \ln a_{k}$ для $k=3, \ldots, \sigma-1$. Так как $a_{k}=f_{1}(k) . f_{2}(k)$, где $f_{1}(k)=$ $2 \sqrt{n-k+1}-1, f_{2}(k)=\exp \left(-k^{2} /(12 n+k)\right)$, то достаточно доказать неравенства $\ln f_{i}(k+1)+\ln f_{i}(k-1)<2 \ln f_{i}(k)$ для $k=3, \ldots, \sigma-1$, $i=1,2$. Для этого достаточно убедиться в справедливости неравенств 
$\left(\ln f_{i}(x)\right)^{\prime \prime}<0$ для $x \in[2, \sigma], i=1,2$, что проверяется просто. Учитывая (10), найдем, что

$$
\begin{aligned}
\frac{a_{2}}{\mu^{2}(2 \sqrt{n-2}-1)^{3}} & =\frac{(2 \sqrt{n-1}-1)^{3}}{(2 \sqrt{n-2}-1)^{5}} y_{n} \\
g_{n} & =\frac{z_{n}}{\sqrt{2 \pi}} \frac{(2 \sqrt{n-2}-1)^{2}}{2 \sqrt{n-1}-1}
\end{aligned}
$$

и затем из формул (5), (7)-(9) полуучим утверждение теоремы.

3 а м е ч а н и е 3 . Верна оценка

$$
Q(n, r, \sigma) \leqslant \min \left(C_{r}^{\sigma} Q_{n, \sigma}, Q_{n, r}\right)
$$

где

$$
Q_{n, t}=\left(g_{n}+1\right)^{t}-2^{-1} t(t-1) g_{n}^{2}-t g_{n}-1 .
$$

Для доказательства достаточно учесть неравенство $C_{\tau}^{k} \leqslant C_{r}^{\sigma} C_{\sigma}^{k}$ при $k=$ $3, \ldots, \sigma$.

3 а м е ч а н и е 4 . Учитывая (5), (6), получим при $\sigma \geqslant 3$ более точную верхнюю оценку суммы $\Sigma(\lambda)$, заменяя $b_{n}$ величиной

$$
\sum_{k=3}^{\sigma} C_{\tau}^{k}(\sqrt{2 \pi})^{-k} \exp \left(-\frac{k^{2}}{12 n+k}\right)(2 \sqrt{n-k+1}-1)^{k-2}
$$

5. Сравнение оценок. Докажем, что при $n \geqslant 2$ оценка величины $\Sigma(0)$ теоремы 3 лучше оценки $\Sigma(0) \leqslant C_{n+r-1}^{n}$ (см. [2]). Случаи $n=$ 2,3 и $n \geqslant r / w$ проверяются просто. Рассмотрим случай $4 \leqslant n<r / w$. Методом индукции по $n$ с использованием равенства $C_{n+r+1}^{r}=C_{n+r}^{r}(r+$ $n+1) /(n+1)$ легко доказать неравенство $C_{n+r}^{r} \geqslant((n+r) b / n)^{n}$, если $n \geqslant 2, r \geqslant \max (2, a n), a>0,0<b \leqslant \min \left(\sqrt{\frac{3}{2}},\left(1+2^{-1} a /(a+1)\right)^{2}\right)$. Полагая $a=0.25<w, b=1.21>h$, убеждаемся, что при $4 \leqslant n<r / w$

$$
C_{n+r}^{r}>\left(\frac{n+r}{n} h\right)^{n}, \quad C_{n+r-1}^{n}>\frac{r}{n+r}\left(\frac{n+r}{n} h\right)^{n}
$$

Приведем пример применения теоремы 3 . Пусть в схеме $\mathbf{c} r$ равновероятными исходами после $n$ испытаний, $4 \leqslant n \leqslant r$, произошло событие $\left(n_{1}, \ldots, n_{r}\right)$ такое, что

$$
\sum_{k=1}^{r} I\left(n_{k} \geqslant t\right)=\nu
$$


где $t$ натуральное. Тогда

$$
\begin{aligned}
& \lambda_{n}=\ln \left(\frac{r}{n}\right)+n^{-1} \sum_{k=1}^{r} \ln \left(n_{k} !\right) \\
& \mathbf{P}_{\mathbf{n}} \leqslant \frac{r}{n+r}\left(\frac{n+r}{n} h\right)^{n} \exp \left(-n \lambda_{n}\right) \leqslant \frac{r}{n+r}\left(\frac{n+r}{r} h\right)^{n}(t !)^{-\nu}
\end{aligned}
$$

Например, при $r=150, n=100, t=4, \nu=25$ (т.е. частоты 25 исходов равны 4, остальные исходы не появились) получим:

$$
\mathbf{P}_{\mathbf{n}} \leqslant \frac{3}{5}\left(\frac{5}{3} h(24)^{-1 / 4}\right)^{100}<1.76 \cdot 10^{-5}
$$

Сравним оценки суммы $\Sigma(\lambda)$ из теорем 3,4 при некоторых соотношениях между $r, n, \sigma$.

Іри фиксированном $\sigma \geqslant 3$ и при $n \rightarrow \infty$ для оценки теоремы 4 имеем

$$
f(n, r, \mathbf{p}, \lambda) \leqslant r+\sqrt{2 \pi n} \pi C_{\tau}^{\sigma}\left(\frac{2 n}{\pi}\right)^{\sigma / 2} \frac{1+\alpha_{n}}{4 n}+\frac{\sqrt{2 \pi n}}{4} r(r-1)
$$

где $\lim _{n \rightarrow \infty} \alpha_{n}=0$. Для всех $n f(n, r, \mathbf{p}, \lambda) \leqslant r+\sqrt{2 \pi n} r(r-1) / 4$ при $\sigma=2, f(n, r, \mathbf{p}, \lambda) \leqslant r$ при $\sigma=1$. Если $n \geqslant r / w$, то оценка теоремы 3 имеет вид $(n / r+1)^{r-1}$ и, следовательно, хуже оценқи теоремы 4 при фиксированном $r$ и $n \rightarrow \infty$. Если $4 \leqslant n<r / w$, то оценка теоремы 3 имеет вид $(1+r / n)^{n} h^{n} r /(n+r)>(1+r / n)^{n} h^{n} w /(w+1)$ и, следовательно, хуже оценки теоремы 4 при фиксированном достаточно большом $n, \sigma \in$ $\{1, \ldots, n-1\}$ и при $r \rightarrow \infty$. Докажем, что оценка теоремы 3 лучше оценки теоремы 4 , если $\sigma=n \geqslant 4$ и

$$
r \geqslant n \frac{1+A_{n, r}}{1-A_{n, r}}-\frac{1}{1-A_{n, r}}
$$

где

$$
\begin{aligned}
& A_{n, r}=\sqrt{2 \pi} h e^{-1} \frac{2 \sqrt{n-1}-1}{(2 \sqrt{n-2}-1)^{2}} D_{n, r} \\
& D_{n, r}=\left(\kappa^{-1} \frac{r}{n+r}(2 \sqrt{n-1}-1)^{-3}(2 \sqrt{n-2}-1)^{5}\right)^{1 / n},
\end{aligned}
$$

$\kappa=\pi \exp \left(-\frac{5}{12}\right) ; 0<A_{n, r}<1$ при $n \geqslant 4$. При $n \geqslant 4$ неравенство $A_{n, r}<B_{n}=\sqrt{2 \pi} h e^{-1}(2 \sqrt{n-3}-1)^{2 / n-1}$ следует из вогнутости функции $\ln (2 \sqrt{x-2}-1)$ на $[3, \infty)$. Так как $B_{n}$ убывает при $n \geqslant 4$ и $B_{5}<1$, то $A_{n, r}<B_{n}<1$ при всех $n \geqslant 5$. Неравенство $A_{4, r}<1$ проверяется вычислением. 
Так как $r \geqslant n, \sigma=n \geqslant 4$, то для функции $f(n, r, \mathbf{p}, \lambda)$ теоремы 4 верны оценки

$$
\begin{aligned}
f(n, r, \mathbf{p}, \lambda) & >\pi \frac{n ! e^{n}}{n^{n}} b_{n}>\pi \frac{n ! e^{n}}{n^{n}} C_{r}^{n} g_{n}^{n} \\
& >\kappa\left(\frac{e(r-n+1)}{\sqrt{2 \pi} n} \frac{(2 \sqrt{n-2}-1)^{2}}{2 \sqrt{n-1}-1}\right)^{n} \frac{(2 \sqrt{n-1}-1)^{3}}{(2 \sqrt{n-2}-1)^{5}}
\end{aligned}
$$

(используем оценки $n ! C_{r}^{n} \geqslant(r-n+1)^{n}, z_{n}^{n}>\exp \left(-\frac{5}{12}\right)$ ). Достаточно доказать, что

$$
\frac{r}{n+r}\left(\frac{n+r}{n} h\right)^{n} \leqslant \kappa\left(\frac{e(r-n+1)}{\sqrt{2 \pi} n} \frac{(2 \sqrt{n-2}-1)^{2}}{2 \sqrt{n-1}-1}\right)^{n} \frac{(2 \sqrt{n-1}-1)^{3}}{(2 \sqrt{n-2}-1)^{5}}
$$

что равносильно неравенству (11).

Получение оценок вероятности $\mathbf{P}_{\mathbf{n}}$ свелось к задаче оценивания вероятности $\mathbf{Q}_{\mathbf{n}}$ события $I(\underline{\mathbf{x}}, \mathbf{p}) \geqslant \lambda_{n}$ (см. доказательство теоремы 1$)$. Из теорем 3,4 работы [7] следует, что

$$
\lim _{n \rightarrow \infty}\left(\mathbf{Q}_{\mathbf{n}} / R_{n}\right)=1
$$

при фиксированном $r$, где

$$
R_{n}=R_{n, 1}=\left(\lambda_{n} n\right)^{(r-3) / 2}\left(\Gamma\left(\frac{r-1}{2}\right)\right)^{-1} \exp \left(-n \lambda_{n}\right)
$$

если $\lim _{n \rightarrow \infty} \lambda_{n}=0, \lim _{n \rightarrow \infty}\left(n \lambda_{n}\right)=\infty$,

$$
R_{n}=R_{n, 2}=d_{n} n^{(r-3) / 2} \exp \left(-n \lambda_{n}\right), \quad d_{n} \in[d, D]
$$

при некоторых неизвестных положительных $d, D, d<D$, если

$$
\lim _{n \rightarrow \infty} \lambda_{n}=\lambda \quad \text { и } \quad \lambda \in\left(0, \frac{p}{2(1-p)}\right) \text {. }
$$

С учетом замечания 1 делаем вывод: при достаточно больших $n$ c вероятностью, близкой к 1 , величина $\lambda_{n}$ близка к 0 при выполнении гипотезы $H(\mathbf{p}, \mathbf{n})$ (поскольку в этом случае n сходится по вероятности к $\mathbf{p}$ ) и близка к $\lambda=I(\mathbf{q}, \mathbf{p})$ при выполнении гипотезы $H(\mathbf{q}, \mathbf{n}), \mathbf{q}=\left(q_{1}, \ldots, q_{r}\right)>$ $(0, \ldots, 0), q_{1}+\cdots+q_{r}=1$, (поскольку в этом случае $\underline{\mathbf{n}}$ сходится по вероятности к q). Таким образом, могут наступать ситуации:

$$
\begin{aligned}
& \mathbf{Q}_{\mathbf{n}} \approx R_{n, 1} \text { при выполнении гипотезы } H(\mathbf{p}, \mathbf{n}), \\
& \mathbf{Q}_{\mathbf{n}} \approx R_{n, 2} \text { при выполненип гипотезы } H(\mathbf{q}, \mathbf{n}) .
\end{aligned}
$$

Заметим, что $2^{-1} p /(1-p)<\gamma_{r}$ при всех достаточно больших $n$ и фиксированном $r$. Действительно, последнее неравенство равносильно 
неравенству

$$
\frac{p}{2(1-p)}+\ln p<u_{n, r}=\ln \left(1-(r-1) n^{-1}\right)-(r-1) n^{-1} \ln (n-r+1),
$$

a $2^{-1} p /(1-p)+\ln p \leqslant(2(r-1))^{-1}-\ln r \leqslant \frac{1}{2}-\ln 2<0$ при $0<p \leqslant 1 / r$, $\lim _{n \rightarrow \infty} u_{n, r}=0$.

Таким образом, при достаточно больших $n$ и когда либо $\lambda_{n}$ близко к $\lambda, 0<\lambda=I(\mathbf{q}, \mathbf{p})<2^{-1} p /(1-p)$, либо $\lambda_{n}$ близко к 0 и величина $n \lambda_{n}$ достаточно велика, с помошью теоремы 4 получаем оценку

$$
\mathbf{Q}_{n} \leqslant\left(\sqrt{\frac{2}{\pi}}\right)^{r-3} n^{(r-1) / 2}\left(1+\alpha_{n, r}\right) \exp \left(-n \lambda_{n}\right)
$$

где $\lim _{n \rightarrow \infty} \alpha_{n, r}=0$, а с помощью теорем 3,4 работы [7] получаем оценку

$$
\mathbf{Q}_{n} \leqslant c_{n, r} n^{(r-3) / 2} \exp \left(-n \lambda_{n}\right)
$$

где $0<c_{1} \leqslant c_{n, r} \leqslant c_{2}<\infty\left(c_{1}, c_{2}\right.$ неизвестны), которая при всех достаточно больших $n$ лучше по крайней мере в $C n$ раз (константа $C$ неизвестна).

6. Оценка вероятности ошибки второго рода при альтернативной гипотезе $H(\mathbf{q}, \mathbf{n})$. Докажем, что критерий $(2)$ позволяет уверенно отклонять гипотезу $H(\mathrm{p}, \mathrm{n})$ при фиксированном $r$ и достаточно больших $n$, когда на самом деле верна гипотеза $H(\mathbf{q}, \mathbf{n}), \mathbf{q}=$ $\left(q_{1}, \ldots, q_{r}\right)>(0, \ldots, 0), q_{1}+\cdots+q_{r}=1, \mathbf{q} \neq \mathbf{p}$, если $\lim _{n \rightarrow \infty} n^{-1} \ln \varepsilon_{n}=$ $\lim _{n \rightarrow \infty} n^{-1} \ln f\left(n, r, \mathbf{p}, \lambda_{n}\right)=0$ (в частности, при $\varepsilon_{n}=\varepsilon>0, f(n, r, \mathbf{p}, \lambda)$ из теорем 3,4$)$. Іри этих условиях с учетом замечания 1 критерий $(2)$ можно представить в виде неравенства

$$
I(\underline{n}, \mathbf{p}) \geqslant \delta_{n}=n^{-1}\left(\ln f\left(n, r, \mathbf{p}, \lambda_{n}\right)-\ln \varepsilon_{n}+M(n, r)-\sum_{k=1}^{r} \varphi\left(n_{k}\right)\right),
$$

$\lim _{n \rightarrow \infty} \delta_{n}=0$

Теорема 5. Если верна гипотеза $H(\mathbf{q}, \mathbf{n})$, по для вероятности $\mathbf{P}$ события $I(\underline{n}, \mathbf{p})<\delta$ при некотором $\delta \in(0, I(\mathbf{q}, \mathbf{p}))$ верна оченка

$$
\mathbf{P} \leqslant \exp (-n I(\mathbf{z}, \mathbf{q}))
$$

2de

$$
\mathbf{z}=\left(z_{1}, \ldots, z_{r}\right) \neq \mathbf{q}, \quad z_{k}=p_{k}^{\tau} q_{k}^{1-\tau}\left(\sum_{i=1}^{r} p_{i}^{\tau} q_{i}^{1-\tau}\right)^{-1}
$$


для $k=1, \ldots, r, \tau-$ - динственभый жа $(0,1)$ хорень уравнения

$$
\begin{aligned}
& F(t)=(t-1) f^{\prime}(t)-f(t)-\delta=0 \\
& f(t)=\ln \sum_{i=1}^{r} p_{i}^{t} q_{i}^{1-t} \quad \partial \Omega s t \in[0,1] .
\end{aligned}
$$

Доказате ль с т в о. Полагая

$$
p_{k}(t)=p_{k}^{t} q_{k}^{1-t}\left(\sum_{i=1}^{r} p_{i}^{t} q_{i}^{1-t}\right)^{-1} \text { при } t \in[0,1]
$$

получим

$$
\begin{aligned}
& F^{\prime}(t)=(t-1) f^{\prime \prime}(t) \\
& f^{\prime \prime}(t)=\sum_{i=1}^{r} p_{i}(t)\left(\ln \left(\frac{p_{i}}{q_{i}}\right)-\sum_{k=1}^{r} p_{k}(t) \ln \left(\frac{p_{k}}{q_{k}}\right)\right)^{2}>0
\end{aligned}
$$

для $t \in[0,1], F(0)=I(\mathbf{q}, \mathbf{p})-\delta>0, F(1)=-\delta<0$. Отсюда следует утверждение о $\tau$.

Имеем

$$
\begin{aligned}
\mathbf{P} & =\sum_{I(\underline{\mathbf{n}}, \mathbf{p})<\delta} \frac{n !}{n_{1} ! \cdots n_{r} !} q_{1}^{n_{1}} \cdots q_{r}^{n_{r}} \\
& =\sum_{I(\underline{\mathbf{n}}, \mathbf{p})<\delta} \frac{n !}{n_{1} ! \cdots n_{r} !} z_{1}^{n_{1}} \cdots z_{r}^{n_{r}} \exp \left(-n \sum_{i=1}^{r} \underline{n}_{i}\left(1+\ln \frac{z_{i}}{q_{i}}\right)+n\right) \\
& \leqslant \exp \left(-n \min _{\mathbf{u} \in U} H(\mathbf{u})\right),
\end{aligned}
$$

где

$$
\begin{aligned}
H(\mathbf{u}) & =\sum_{i=1}^{r} u_{i}\left(1+\ln \frac{z_{i}}{q_{i}}\right)-1 \\
U & =\left\{\mathbf{u}=\left(u_{1}, \ldots, u_{r}\right): u_{1} \geqslant 0, \ldots, u_{r} \geqslant 0,-\sum_{i=1}^{r} u_{i}+1 \geqslant 0,\right. \\
& \left.\sum_{i=1}^{r} u_{i}-1 \geqslant 0,-I(\mathbf{u}, \mathbf{p})+\delta \geqslant 0\right\} .
\end{aligned}
$$

Положим

$$
\beta_{1}=0, \quad \beta_{2}=\frac{1-f(\tau)}{1-\tau}>0, \quad \beta_{3}=\frac{\tau}{1-\tau}>0
$$


Точка z удовлетворяет следуюшей системе неравенств и уравнений: $z_{1}>$ $0, \ldots, z_{r}>0$,

$$
\begin{aligned}
-\sum_{i=1}^{r} z_{i} & +1=0, \quad \sum_{i=1}^{r} z_{i}-1=0, \quad-I(\mathbf{z}, \mathbf{p})+\delta=0 \\
\left.\frac{\partial H(\mathbf{u})}{\partial u_{j}}\right|_{\mathbf{u}=\mathbf{z}}= & \left.\beta_{1} \frac{\partial}{\partial u_{j}}\left(-\sum_{i=1}^{r} u_{i}+1\right)\right|_{\mathbf{u}=\mathbf{z}} \\
& +\left.\beta_{2} \frac{\partial}{\partial u_{j}}\left(\sum_{i=1}^{r} u_{i}-1\right)\right|_{\mathbf{u}=\mathbf{z}}+\left.\beta_{3} \frac{\partial(-I(\mathbf{u}, \mathbf{p})+\delta)}{\partial u_{j}}\right|_{\mathbf{u}=\mathbf{z}}
\end{aligned}
$$

для $j=1, \ldots, r$. Применяя достаточное условие глобального минимума основной задачи выпуклого программирования (см., например, теорему 3.5 .1 в $[8$, с. $53,43-45])$, найдем, что

$$
\min _{\mathbf{u} \in U} H(\mathbf{u})=H(\mathbf{z})=I(\mathbf{z}, \mathbf{q}) .
$$

Заметим, что при $\mathbf{p} \neq \mathbf{q}$ верны неравенства $\mathbf{z} \neq \mathbf{q}, I(\mathbf{z}, \mathbf{q})>0$.

\section{СПИСОК ЛИТЕРАТУРЫ}

1. Кокс Д., Хиякли Д. Задачи по теоретической статистике с решениями. М.: Мир, $1981,224 \mathrm{c}$.

2. Hoeffding $W$. Asymptotically optimal tests for multinomial distributions. - Ann. Math. Statist., 1965, v. 36, № 2, p. 369-401.

3. Феллер В. Введение в теорию вероятностей и ее приложения, т. 1. М.: Мир, 1984, $527 \mathrm{c}$.

4. Сачков B. H. Комбинаторные методы дискретной математики. М.: Наука, 1977, $319 \mathrm{c}$.

5. Риордан Дж. Комбинаторные тождества. М.: Наука, 1982, 255 с.

6. Яблонский C. B., Лупанов О. Б. (ред.) Дискретная математика и математические вопросы кибернетики, т. 1. М.: Наука, 1974, 311 с.

7. Саяов И. Н. Способ получения асимптотики вероятности больших уклонений нелинейных статистик от мультиномиального распределения и его применения. Теория вероятн. и ее примен., 1965, т. Х, в. 4, с. 761-763.

8. Карманоя В. Г. Математическое программирование. М.: Наука, 1980, 256 с.

Поступила в редакцию 12.V.1996 